\title{
Unified Template Strategy for Editing Multiple Remote C-H Bonds
}

Authors: Zhoulong Fan,,${ }^{1,}$ Xiangyang Chen,,${ }^{2 \dagger}$ Keita Tanaka, ${ }^{1}$ Han Seul Park, ${ }^{1}$ Nelson Y. S. Lam, ${ }^{1}$ Jonathan J. Wong, ${ }^{2}$ K. N. Houk, ${ }^{2, *}$ Jin-Quan $\mathrm{Yu}^{1, *}$

Affiliations: ${ }^{1}$ Department of Chemistry, The Scripps Research Institute, 10550 North Torrey Pines Road, La Jolla, California 92037, United States. ${ }^{2}$ Department of Chemistry and Biochemistry, University of California, Los Angeles, California 90095, United States

*Correspondence to: yu200@scripps.edu; houk@chem.ucla.edu

$\dagger$ These authors contributed equally to this work.

Abstract: Through consecutive selective $\mathrm{C}-\mathrm{H}$ functionalization at multiple sites, the direct molecular editing of heteroarene carbon-hydrogen $(\mathrm{C}-\mathrm{H})$ bonds has the potential to grant rapid access into diverse molecular space; a valuable but often challenging venture to achieve in medicinal chemistry. Contrasting with electronically-biased heterocyclic $\mathrm{C}-\mathrm{H}$ bonds, remote benzocyclic $\mathrm{C}-\mathrm{H}$ bonds on bicyclic aza-arenes are especially difficult to differentiate due to lack of intrinsic steric/electronic biases. Through careful consideration of distance and geometric parameters, we herein report a unified catalytic directing template strategy that enables the modular functionalization of chemically-similar and adjacent remote positions on bicyclic aza-arene scaffolds. Differentiated by using two structurally distinct catalytic directing templates, this method enables direct $\mathrm{C}-\mathrm{H}$ olefination, alkynylation, and allylation at previously inaccessible $\mathrm{C} 6$ and $\mathrm{C} 7$ positions of quinolines, and is amenable to the iterative, 
modular, and late-stage $\mathrm{C}-\mathrm{H}$ editing of quinoline-containing pharmacophores and pharmaceuticals. This report, in combination with our previous C5-selective template and other complementary methods, now fully establishes a unified 'molecular editing' strategy to directly modify aza-arene heterocycles at any given site.

Main Text: The efficient generation of diverse structural analogues forms a perennial synthetic challenge that underpins drug discovery. For a given molecular scaffold or pharmacophore, the selective and iterative activation of inert $\mathrm{C}-\mathrm{H}$ bonds represents the most direct strategy for the expeditious generation of structural diversity ${ }^{1,2}$. For example, functionalization with ten coupling partners at four different sites of quinoline scaffold could generate up to 10,000 structurally unique analogues using a unified editing strategy, which can be challenging to achieve using de novo synthesis (Fig. 1a). This notionally ideal 'molecular editing' approach, however, is marred by a lack of reliable methods for their selective functionalization, curtailing broader realization in a translational context. ${ }^{3}$ As a privileged pharmacophore for diverse biological targets, aza-arene heterocycles are particularly dominant within the realm of drug discovery. Within the azine component, leveraging a substrate's intrinsic electronic properties have enabled the now-established site-selective functionalizations of $\mathrm{C} 2^{4-6} / \mathrm{C} 4-\mathrm{H}^{7-10}$ under a nucleophilic metallation regimen, and at $\mathrm{C} 3-\mathrm{H}^{11,12}$ via the corresponding electrophilic metallation process. Comparatively, selective functionalization of $\mathrm{C}-\mathrm{H}$ bonds on the benzocyclic component of bicyclic aza-arene heterocycles have remained underexplored. For these chemically-similar $\mathrm{C}-\mathrm{H}$ bonds, leverage of proximity-driven effects to selectively direct the catalyst has been limited to benzocyclic $\mathrm{C} 8-\mathrm{H}$ bonds 
adjacent to Lewis basic heteroatoms ${ }^{13-17}$. Notably, the selectively editing of remote positions, such as $\mathrm{C} 5-\mathrm{C} 7$ on quinoline-type scaffolds, remain inaccessible to the established methods described above (Fig. 1a).

We surmised that this eminent problem could be solved using reversiblybinding directing templates capable of selectively positioning the catalyst proximate to a target remote $\mathrm{C}-\mathrm{H}$ bond. More specifically, we conjectured that fine-tuning distance and geometric parameters of the macrocyclic pre-transition state arising from catalyst coordination could favor placement of the catalyst near the desired $\mathrm{C}-\mathrm{H}$ bond over others, thus resulting in selective activation ${ }^{18-24}$. In this context, bicyclic aza-arene scaffolds pose further obstacles for template-directed remote regioselection; in addition to suppressing functionalization at activated $(\mathrm{C} 2-\mathrm{C} 4)$ sites, the multiple adjacent, yet minutely inequivalent and unactivated remote benzocyclic positions (C5-C7) demand stringent spatial precision for their discrimination and selective activation. The feasibility of this template-directed approach for the activation of remote benzocyclic C-H bonds was first indicated in 2017, where stoichiometric template loadings have enabled the singular C5-functionalization of quinoline $\mathrm{e}^{25-26}$. We also combined C5selective template with a norbornene relay strategy to realize C6-selective arylation ${ }^{27}$; this strategy requires a vacant $\mathrm{C} 5$ position to enable its successful C6-functionalization. However, executing a template strategy to access C6 and C7 positions is confounded by their high degree of spatial similarity relative to the anchoring $N$ atom that challenges precise template design. Additionally, the direct activation of $\mathrm{C} 6$ and $\mathrm{C} 7$ positions, as compared with the marginally more nucleophilic C5 and C8 positions, is particularly 
difficult both for the chemical inertness and electronic similarity of the two $\mathrm{C}-\mathrm{H}$ bonds - an observation that is correlated to their lower relative HOMO coefficients (Fig. 1b). Altogether, the development of new catalytic templates, as well as a more practical method for their deployment, is required to realize the direct activation of bicyclic azaarenes at these remaining unexplored $\mathrm{C}-\mathrm{H}$ bonds; the success of which is essential for achieving full-range and sequential molecular editing of heterocycles in any desired order (Fig. 1c).

Driven to develop this unprecedented synthetic tool, we herein report the successful design of two catalytic directing templates for the site-selective C6 and C7$\mathrm{H}$ activation of bicyclic aza-arenes, thereby realizing the differentiation of three consecutive chemically-similar $\mathrm{C}-\mathrm{H}$ bonds in conjunction with our previously developed C5-selective template. The catalytic pyridine-based template recruits the aza-arene substrate through $\mathrm{N}$-coordination, enabling the directing arm to deliver the catalyst and precisely activate a single target $\mathrm{C}-\mathrm{H}$ bond. Careful adjustment of the template distance and geometry relative to the template anchoring point enables the differentiation and selective activation at either the C6 or C7 position (Fig. 1d). In parallel, we discovered that the use of a simple and readily prepared template chaperone (TC) can turn over the directing template, therefore allowing it to be used catalytically for the first time. This method is amenable to the iterative $\mathrm{C}-\mathrm{H}$ editing of pharmacophore and a late-stage context for a variety of bicyclic aza-arene-containing drugs, facilitating the direct and selective functionalization at these positions not currently accessible by known reactions. 

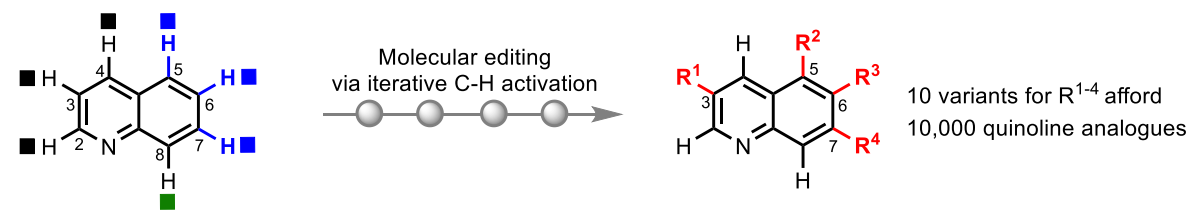

Selectivity based on electronic effects ${ }^{4-12}$ Selectivity based on proximity ${ }^{13-17}$

Selectivity requires template directivity ${ }^{25-27}$

b Reactivity challenges for $\mathrm{C} 6$ and $\mathrm{C} 7$ positions (HOMO analysis)

C Proposed catalytic directing template strategy
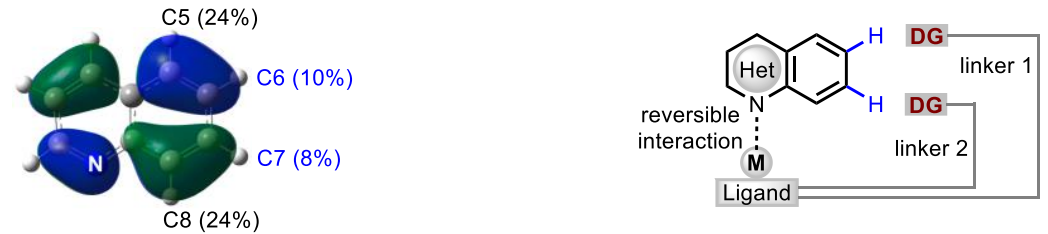

d $\mathrm{C} 6$ and C7-selective templates based on distance and geometric considerations (This work)

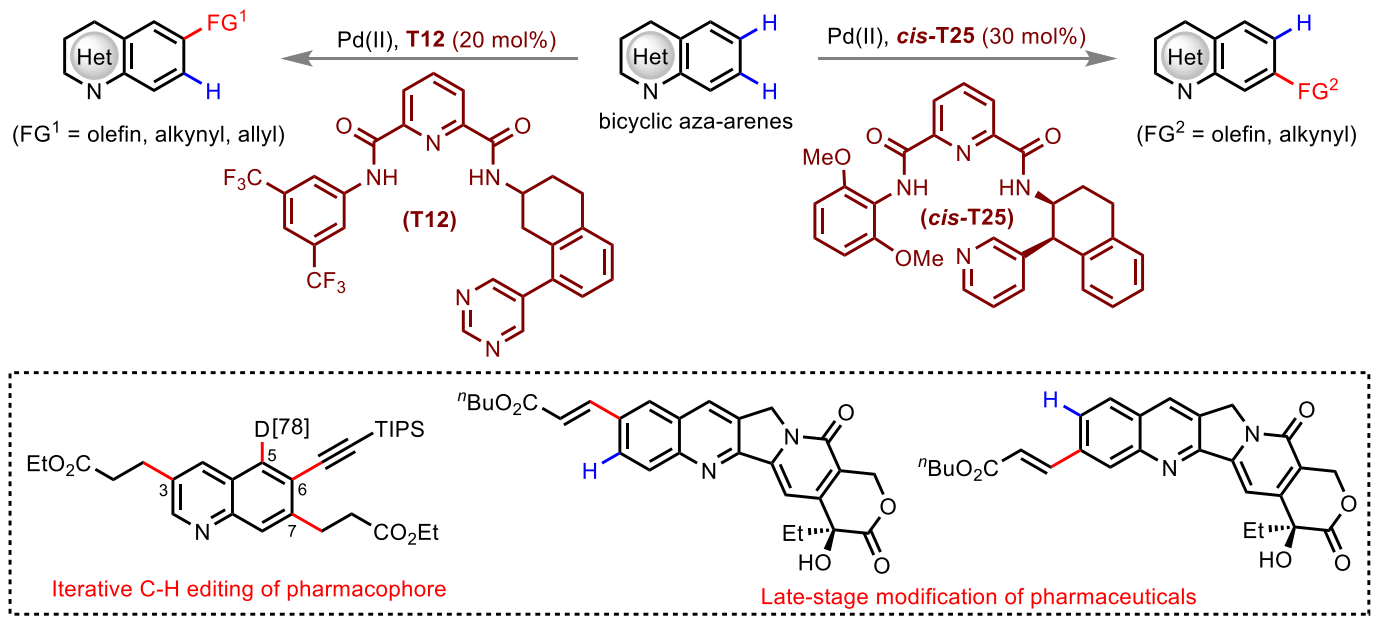

Fig. 1. Molecular editing of heterocycles. a, Molecular editing of quinoline. b,

Selectivity challenges for $\mathrm{C} 6$ and $\mathrm{C} 7$ positions (HOMO analysis). $(\%)=$ atomic contributions to the HOMO under the level of M06/6-31G(d). c, Proposed catalytic directing template strategy. d, C6 and C7-selective templates based on distance and geometric considerations (This work). M, metal; DG, directing group; FG, functional group.

Using quinoline 1a as the model substrate and template chaperone TC8 (two steps, no chromatography), we initially targeted the development of a selective $\mathrm{C} 6-\mathrm{H}$ olefination reaction. Considering that our previous C5 template is used in stoichiometric amounts, a key objective at the outset of our studies was the catalytic 
use of our templates. Therefore, we introduced easily synthetized symmetrical template chaperone TC8 to mask the quinoline nitrogen and facilitate product turnover from the directing template. An initial hit was found through the use of 2-fluoro-3-phenylpyridyl motif as the directing group (T2, see Supplementary Information, Table S1) ${ }^{28}$. Systematic screen of linker length ( $\mathbf{T 1}$ to $\mathbf{T 3}$, Table S1) revealed that a two-carbon linker was optimal (T3, Table S1) We noticed that rigidifying the linker between the template scaffold and directing motif (T7, Table S1) improved the reactivity, though the rigid four-carbon phenyl linker pushed the selectivity towards the undesired C5 position. Taking these observations together, a rigidified analogue of T3 bearing an alicyclic two-carbon spacer (T8) was next pursued, which to our delight, gave marked improvement both yield and C6 selectivity. Further tuning of the directing motif (T9 to T11, Table S1) showed that yields were improved in T11, which guided our choice towards switching from pyridyl to pyrimidyl directing group (T12), giving improved the yield and selectivity (Fig. 2a). A subsequent examination on the left-hand portion of the template (T13 to T15, Table S1) and template chaperone scaffolds (TC1 to TC7, TC9 to TC12, Table S1) gave no noticeable improvement in both reactivity and selectivity. The optimal result with $\mathbf{T 1 2}$ and $\mathbf{T C 8}$, both bearing 3,5ditrifluoromethylphenyl side arms, likely arises from their structural homology, improving the efficacy of substrate/product exchange in the reaction. In all cases, the incorporated palladium within the directing template and template chaperones can be easily recovered as the TC-Pd-MeCN complex and recycled with no loss in reaction efficacy (see Supplementary Information 2.8). 
With optimized template and conditions in hand, we next evaluated the reaction scope with respect to quinoline and related heterocycles (2a to 2ab, Fig. 2b). Various electron-donating and electron-withdrawing groups on quinoline were compatible in the reaction, showing little difference in yield or selectivity and confirming the power of our proximity-driven directing approach to override inherent electronic preferences. In contrast, the reactivity and C6-selectivity of the reaction was sensitive to steric hindrance arising from a substituent's position on the heterocyclic scaffold (2a to $2 \mathbf{r}$ ). A series of multiply substituted quinolines were also well tolerated (2s to $\mathbf{2 w}$ ). We were pleased to find that the reaction also tolerates a variety of bicyclic and polycyclic azaarenes; quinoxaline ( $\mathbf{2 x}$ ), benzothiophene (2y) and polycyclic quinolines ( $\mathbf{2 z}$ to $\mathbf{2} \mathbf{a b})$ all afforded the desired products in good yield and high selectivity. Next, we examined the scope of coupling partners with unsubstituted quinoline under the standard conditions. A variety of acrylate (2ac to 2ai), vinylamide (2aj and 2ak), vinylsulfone (2al), vinylphosphonate (2am), styrene (2an) and more complex terpenoid-derived acrylate coupling partners (2ao, from menthol; 2ap, from tetrahydrogeraniol) were well tolerated, delivering the corresponding products in good to excellent yield and selectivity.

Next, detailed experimental and computational studies were conducted to understand the origin of C6-selectivity in our catalytic templates. Density functional theory (DFT) analysis revealed that $\mathrm{C}-\mathrm{H}$ activation step was not turnover-limiting, consistent with observed kinetic isotope effects studies (see Supplementary Information 2.14). While inspection of the modelled substrate-template complex revealed that the 
C7 position was closest to the template nitrogen atom responsible for catalyst recruitment, analysis of the concerted metalation deprotonation (CMD) step immediately excluded a C7-selective pathway because of higher energies incurred by repulsive interactions between the template phenyl ring and ligand acetyl groups (Fig. 2c). The analysis also suggested that the initial $\mathrm{C}-\mathrm{H}$ metalation was likely unselective between C5 and C6, which was corroborated through observing unselective deuterium incorporation (see Supplementary Information 2.13). Further analysis of their respective downstream pathways revealed a lower overall free energy profile for the C6-selective alkene insertion step relative to $\mathrm{C} 5$ due to larger template distortion required to access the $\mathrm{C} 5$ position. Therefore, a combination of a more favored $\mathrm{C}-\mathrm{H}$ activation (disfavoring C7) and alkene insertion steps (disfavoring C5) give rise to the observed C6 selectivity for this template. 


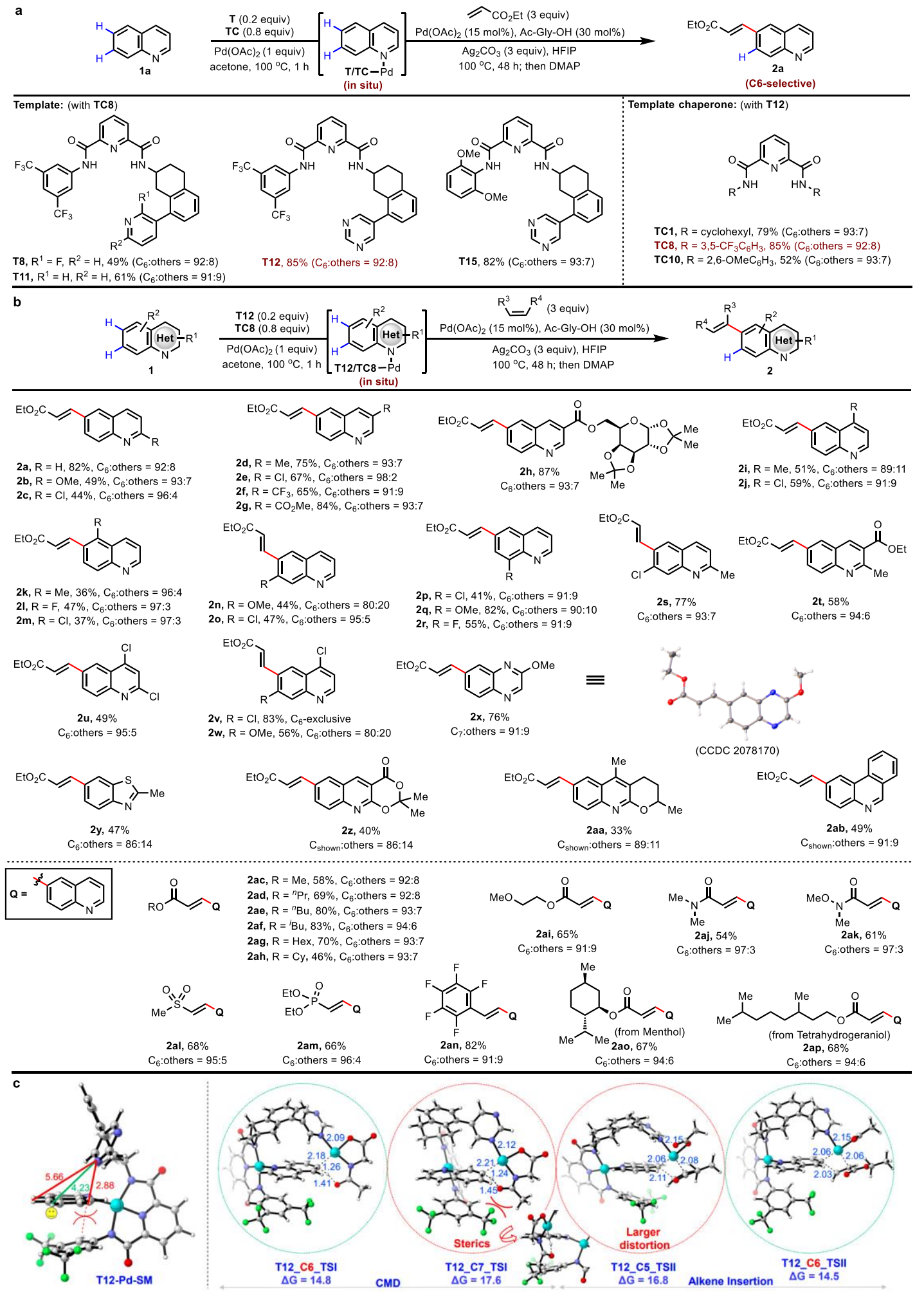

Fig. 2. C6 (and related) selective $\mathrm{C}-\mathrm{H}$ olefination reactions of quinoline and related

heterocycles. a, Selected optimization of directing template and template chaperone scaffolds. The yield and ratio ( $\mathrm{C}_{6}$ :others) of $\mathbf{2 a}$ were determined by ${ }^{1} \mathrm{H}$ NMR analysis. b, The scope of bicyclic aza-arenes and olefins. For each entry, data are reported as 
isolated yields. The ratio $\left(\mathrm{C}_{6}\right.$ :others or $\mathrm{C}_{\text {shown}}$ :others) of olefination products was determined by ${ }^{1} \mathrm{H}$ NMR analysis using 1,3,5-trimethoxybenzene as the internal standard. Conditions for $\mathbf{2 u}$ : T8 (0.2 equiv). Conditions for $\mathbf{2 x}$ and $\mathbf{2 a b}$ : T15 (0.2 equiv) and TC10 (0.8 equiv). c, DFT analysis rationalizes the observed C6 selectivity for template T12. Bond lengths are denoted in $\AA$.

The success of our reversible C6-selective template prompted us to investigate whether C7-selectivity was also feasible under this catalytic template strategy through judicious optimization of distance and geometry. Noting that the C7 differs from C6 by a single $\mathrm{C}-\mathrm{C}$ bond on the quinoline skeleton, we initially prepared templates bearing a 2-phenylpyridyl directing group T17 (see Supplementary Information, Table S2) and truncated alicyclic linker T18 (Table S2). In both cases, no more than trace amounts of olefinated product was detected. An extensive survey of the template scaffold space identified T19 as a hit, notably omitting the 2-phenyl group from the best C6-selective template (T12). Yield and selectivity further improved when a two-carbon spacer was positioned between the template scaffold and the directing 3-pyridyl motif (T20, Table S2). Surmising that increased rigidity of the directing arm might confer heightened reactivity and selectivity, templates bearing more rigid two-carbon spacers (T21 to T24) were prepared. In particular, we noted that both cis- and trans-T24 delivered the product 3a in moderate yield and high selectivity. Systematic tuning of the template's left arm identified that a 2,6-dimethoxyphenyl motif provided markedly improved C7 yield and selectivity, with the best C7-selective template (cis-T25) afforded 3a in 67\% NMR yield with 96:4 selectivity (Fig. 3a). Additional control studies confirmed the importance of each aforementioned structural feature: modifying the relative 
configuration (trans-T25), the template's left arm to other 2,(4),6-substitutions (T26 and T27, Table S2), as well as the use of a pyrimidyl directing group (T28, analogue of T15, Table S2), all deleteriously impacted yield and C7 selectivity. Further optimization indicated that racemic $N$-acetyl-phenylalanine (Ac- $D L-\mathrm{Phe}-\mathrm{OH}$ ) performed as the best ligand in this C7-selective system, ascribed to differential matched reactivities and selectivities of enantiopure cis-T25 with each enantiomer of Ac- $D L-P h e-O H$ (see Supplementary Information, Table S2).

Under optimized C7-selective conditions, a variety of bicyclic aza-arenes were examined (Fig. 3b). A range of C3 and C4-substituted quinolines smoothly afforded the C7-olefinated products (3a to $\mathbf{3 k}$ ) in good yield and excellent selectivity. Treating C5, C6-substituted and unsubstituted quinolines interestingly provided a readily separable mixture of $\mathrm{C} 7$ and $\mathrm{C} 3$ olefinated products that typically favored the $\mathrm{C} 7$ position. This outcome was ascribed to similar spatial distance and geometry of C3 and C7 to the directing arm of template cis-T25; an observation supported by DFT calculations with quinoline 1a (both of C3 and C7 gave lower energies than C5 and C6 in the energy profile, see Supplementary Information, Fig. S12). Other pharmaceutically important heterocycles, such as quinoxaline, benzothiophene, and phenanthridine were also compatible, generating distally-olefinated products $\mathbf{3 o}$ to $\mathbf{3 q}$ in a site-selective manner. Additionally, a diverse set of olefinic coupling partners were competent using $\mathbf{1 d}$ as the substrate, successfully reacting with acrylates ( $\mathbf{3 r}$ to $\mathbf{3 u}, \mathbf{3 y}$ and $\mathbf{3 z}$ ), vinyl sulfone (3v), vinyl phosphonate $(\mathbf{3 w})$ and styrene $(\mathbf{3 x})$ to give the corresponding products in moderate to good yields with excellent C7-selectivity. To rationalize the observed 
selectivity, DFT analysis was conducted for directing template cis-T25 with quinoline 1d, which showed C7 closest to the template nitrogen responsible for catalyst recruitment (Fig. 3c). Contrasting with the C6-template, cis-T25_C7 transition states gave the lowest energies for all key steps in comparison to other positions (see Supplementary Information, Fig. S8). Further inspection revealed that increased template distortion was required to access both $\mathrm{C} 5$ and $\mathrm{C} 6$ positions, leaving $\mathrm{C} 7$ as the sole favorable pathway for this template. 


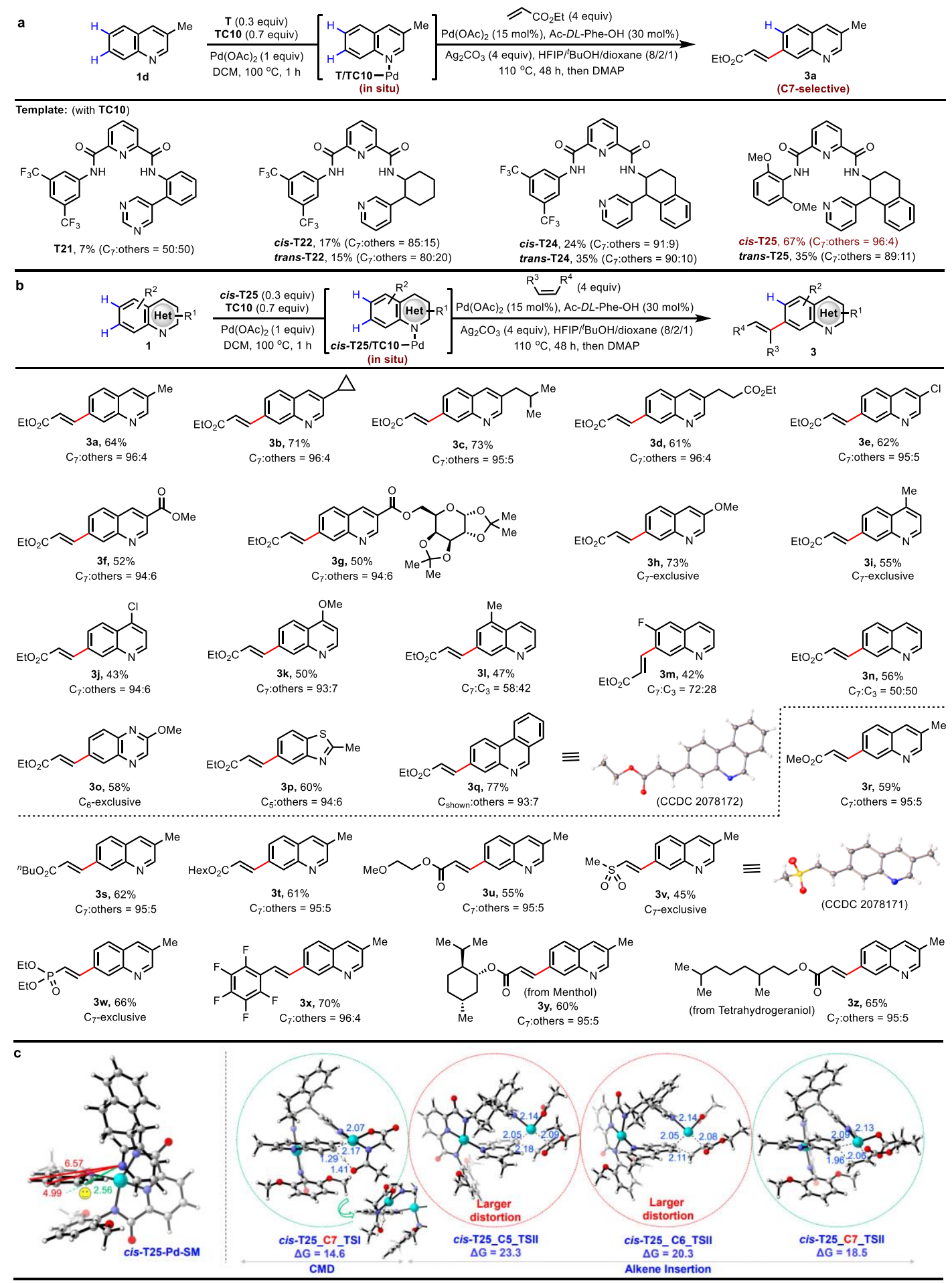

Fig. 3. C7 (and related) selective $\mathrm{C}-\mathrm{H}$ olefination reactions of quinoline and related

heterocycles. a, Selected directing template optimization. The yield and ratio ( $\mathrm{C}_{7}$ :others) of 3a were determined by ${ }^{1} \mathrm{H}$ NMR analysis. $\mathbf{b}$, Scope of bicyclic aza-arenes and olefins. For each entry, data are reported as isolated yields. The ratio $\left(\mathrm{C}_{7}\right.$ :others or $\mathrm{C}_{\text {shown}}$ :others) of olefination products was determined by ${ }^{1} \mathrm{H}$ NMR analysis using 1,3,5- 
trimethoxybenzene as the internal standard. c, DFT analysis rationalizes the observed C7 selectivity for template cis-T25. Bond lengths are denoted in $\AA$.

The scope of transformation that can be intercepted from this catalytic remotedirecting template was broadened through the development of remote site-selective $\mathrm{C}-$ $\mathrm{H}$ alkynylation and allylation of aza-arenes (Fig. 4a, $4 \mathbf{a}$ to $4 \mathbf{h}$; $5 \mathbf{a}$ to $\mathbf{5 f}$; $6 \mathbf{a}$ to $6 \mathbf{e}$ ); representing versatile linchpins for further diversification ${ }^{29-31}$. Uniformly, high siteselectivity was obtained for the template-assisted C6 and C7-selective alkynylation reactions; in some cases, obtaining exclusive selectivity for alkynylated products $(\mathbf{4 c}$, $\mathbf{4 d}, \mathbf{4 f}$, and $\mathbf{4 g}$ ). The corresponding allylation reactions were similarly effective (5a to 5f), giving comparatively higher reactivity albeit with slightly reduced C6-selectivity. In all cases, a range of substitutions were tolerated, signaling the robustness of this catalytic template strategy for direct remote functionalization. 


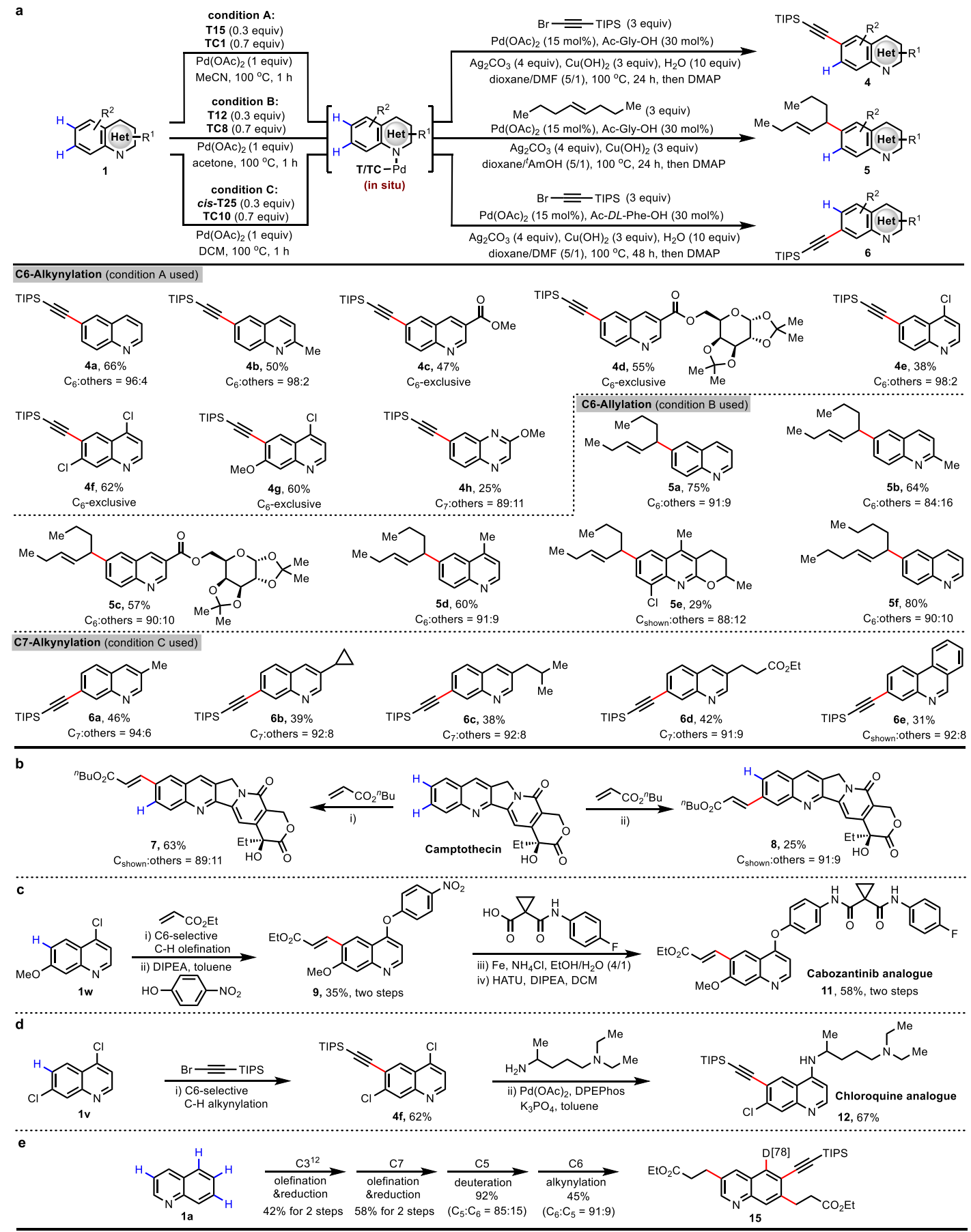

Fig. 4. Other transformations and synthetic applications. a, Site-selective $\mathrm{C}-\mathrm{H}$ alkynylation and allylation of quinoline and related heterocycles. b, Late-stage remote site-selective $\mathrm{C}-\mathrm{H}$ modification of camptothecin. Conditions for $\mathbf{5 f}$ : trans-5-decene (3 equiv). For late-stage modification of camptothecin, reaction conditions: i) T15 (0.2 equiv), TC10 (0.8 equiv), $\mathrm{Pd}(\mathrm{OAc})_{2}\left(1\right.$ equiv), acetone, $100{ }^{\circ} \mathrm{C}, 1 \mathrm{~h}$; then $\mathrm{Pd}(\mathrm{OAc})_{2}(15$ mol\%), Ac-Gly-OH (30 mol\%), $\mathrm{Ag}_{2} \mathrm{CO}_{3}$ (3 equiv), HFIP, $100{ }^{\circ} \mathrm{C}, 48 \mathrm{~h}$; then DMAP. 
ii) $c i s-\mathbf{T} 25$ ( 0.2 equiv), TC1 ( 0.8 equiv), $\mathrm{Pd}(\mathrm{OAc})_{2}$ ( 1 equiv), acetone, $100{ }^{\circ} \mathrm{C}, 1 \mathrm{~h}$; then $\mathrm{Pd}(\mathrm{OAc})_{2} \quad(15 \mathrm{~mol} \%), \quad \mathrm{Ac}-D L-\mathrm{Phe}-\mathrm{OH} \quad(30 \mathrm{~mol} \%), \quad \mathrm{Ag}_{2} \mathrm{CO}_{3} \quad(4$ equiv $)$, $\mathrm{HFIP} /{ }^{t} \mathrm{BuOH} /$ dioxane $(8 / 2 / 1), 110^{\circ} \mathrm{C}, 48 \mathrm{~h}$; then DMAP. c, Synthesis of cabozantinib analogue through C6-H olefination. d, Synthesis of chloroquine analogue through C6$\mathrm{H}$ alkynylation. e, Molecular editing of quinoline achieved through iterative $\mathrm{C}-\mathrm{H}$ activation. Detailed reaction conditions are provided in the Supplementary Information 2.12. Deuterium incorporation is shown in square brackets.

The applicability of this method in a drug discovery context was first exemplified by the divergent late-stage site-selective $\mathrm{C}-\mathrm{H}$ functionalization of the anticancer natural product camptothecin ${ }^{32}$ (Fig. 4b). Subjecting camptothecin to our optimized C6-selective catalytic template generated novel analogue 7 in $63 \%$ yield, while the corresponding C7-selective catalytic template generated its regioisomer $\mathbf{8}$ in $25 \%$ yield. Furthermore, successful direct $\mathrm{C}-\mathrm{H}$ editing of key pharmacophores was also demonstrated, providing expedient access to novel building blocks and analogues of known biologically active molecules. For example, 1w underwent C6-selective olefination to give $\mathbf{2 w}$, which could be elaborated following substitution to deliver $\mathbf{9}$, an analogue of the key intermediate of cabozantinib (treatment against medullary thyroid and renal carcinoma) $)^{33,34}$. From 9, novel cabozantinib derivative $\mathbf{1 1}$ was prepared in 58\% isolated yield over two steps through reduction and subsequent amidation reaction. Likewise, conversion of $\mathbf{1 v}$ to $\mathbf{4 f}$ under our C6-selective alkynylation conditions followed by regioselective Buchwald-Hartwig amination afforded an alkynylated chloroquine analogue $12^{35}$. Both transformations enable the ready access of otherwise challenging to synthesize bicyclic aza-arenes bearing unusual 
substitution patterns, which can enable the facile synthesis of diverse compound libraries for ongoing pharmaceutical development. Finally, we were eager to assess the complementarity of our new templates to molecularly edit any site on a quinoline scaffold; its feasibility was demonstrated in the successful iterative $\mathrm{C}-\mathrm{H}$ activation at C3, C7, C5 and C6-H to access product $\mathbf{1 5}$ bearing diverse substitutions.

In summary, a unified catalytic remote-directing template strategy was developed to enable the divergent Pd-catalyzed remote $\mathrm{C} 6$ and $\mathrm{C} 7-\mathrm{H}$ activations of a range of pharmaceutically-relevant bicyclic aza-arene scaffolds. The modularity of C6/C7 functionalization described herein complements our C5-selective method, which taken altogether, completes the suite of reactions required to directly edit all $\mathrm{C}-\mathrm{H}$ bonds in bicyclic aza-arene scaffolds in an iterative manner. The generality of this template approach was also broadened to include a series of remote alkynylation and allylations. Computational rationalization of how subtle differences in distance and geometry of the templates can alter site-selectivity lends confidence for the future design of catalytic templates for other classes of substrates. In doing so, this unified template strategy validates the power and potential of carefully designed directing templates for editing remote $\mathrm{C}-\mathrm{H}$ bonds at multiple sites of pharmacophores. We anticipate that further development could enable a toolkit of catalytic directing templates able to selectively target any bond within a complex molecule at-will, enabling the facile construction and derivation of molecular scaffolds otherwise circuitous using canonical techniques. 
Acknowledgements: We gratefully acknowledge The Scripps Research Institute and the NIH (National Institute of General Medical Sciences grant R01 GM102265) for their financial support. Computations were performed on the Hoffman2 cluster at UCLA and the Extreme Science and Engineering Discovery Environment (XSEDE), which is supported by the National Science Foundation (OCI-1053575). We are grateful for financial support of the UCLA work from the National Science Foundation (USA) (CHE-1764328 to KNH) and the National Science Foundation (USA) under the NSF Center for Selective C-H Functionalization (CHE-1700982). Dr. Jason Chen, Brittany Sanchez, and Emily Sturgell are acknowledged for their assistance with LCMS analysis. We thank Dr. Jake Bailey and the UCSD Crystallography Facility for Xray crystallographic analysis.

Author contributions: Z. F. developed the templates, optimized the conditions, and investigated the substrate scope. X. C. and J. W. performed the computational studies. Z. F. and K. T. developed the template chaperones. H. S. P. and N. Y. S. L. prepared the substrates and acrylates. J.-Q. Y., K. N. H., Z. F., and N. Y. S. L. prepared the manuscript. J.-Q. Y. directed the project.

Competing interests: The authors declare no competing interests.

Supplementary Information is available in the online version of the paper.

Data availability: The data supporting the findings of this study are available within the article and its Supplementary Information files. 


\section{References:}

1 Yamaguchi, J., Yamaguchi, A. D. \& Itami, K. C-H bond functionalization: emerging synthetic tools for natural products and pharmaceuticals. Angew. Chem. Int. Ed. 51, 8960-9009 (2012).

2 Lam, N. Y. S., Wu, K. \& Yu, J.-Q. Advancing the logic of chemical synthesis: $\mathrm{C}-\mathrm{H}$ activation as strategic and tactical disconnections for $\mathrm{C}-\mathrm{C}$ bond construction. Angew. Chem. Int. Ed. 60, 15767-15790 (2021).

3 Campos, K. R. et al. The importance of synthetic chemistry in the pharmaceutical industry. Science 363, eaat0805 (2019).

4 Berman, A. M., Lewis, J. C., Bergman, R. G. \& Ellman, J. A. Rh(I)-catalyzed direct arylation of pyridines and quinolines. J. Am. Chem. Soc. 130, 1492614927 (2008).

5 Nakao, Y., Kanyiva, K. S. \& Hiyama, T. A strategy for C-H activation of pyridines: direct C-2 selective alkenylation of pyridines by nickel/lewis acid catalysis. J. Am. Chem. Soc. 130, 2448-2449 (2008).

6 Guan, B.-T. \& Hou, Z. Rare-earth-catalyzed C-H bond addition of pyridines to olefins. J. Am. Chem. Soc. 133, 18086-18089 (2011).

7 Nakao, Y., Yamada, Y., Kashihara, N. \& Hiyama, T. Selective C-4 alkylation of pyridine by nickel/lewis acid catalysis. J. Am. Chem. Soc. 132, 13666-13668 (2010).

8 Tsai, C.-C. et al. Bimetallic nickel aluminun mediated para-selective alkenylation of pyridine: direct observation of $\eta^{2}, \eta^{1}$-pyridine $\mathrm{Ni}(0)-\mathrm{Al}(\mathrm{III})$ intermediates prior to $\mathrm{C}-\mathrm{H}$ bond activation. J. Am. Chem. Soc. 132, $11887-$ 11889 (2010).

9 Chen, Q., du Jourdin, X. M. \& Knochel, P. Transition-metal-free BF B-mediated $^{2}$ regioselective direct alkylation and arylation of functionalized pyridines using Grignard or organozinc reagents. J. Am. Chem. Soc. 135, 4958-4961 (2013).

10 Yamamoto, S., Saga, Y., Andou, T., Matsunaga, S. \& Kanai, M. Cobaltcatalyzed C-4 selective alkylation of quinolines. Adv. Synth. Catal. 356, 401405 (2014).

11 Takagi, J., Sato, K., Hartwig, J. T., Ishiyama, T. \& Miyaura, N. Iridiumcatalyzed $\mathrm{C}-\mathrm{H}$ coupling reaction of heteroaromatic compounds with bis(pinacolato)diboron: regioselective synthesis of heteroarylboronates. Tetrahedron Lett. 43, 5649-5651 (2002).

12 Ye, M., Gao, G.-L. \& Yu, J.-Q. Ligand-promoted C-3 selective C-H olefination of pyridines with Pd catalysts. J. Am. Chem. Soc. 133, 6964-6967 (2011). 
13 Kwak, J., Kim, M. \& Chang, S. Rh(NHC)-catalyzed direct and selective arylation of quinolines at the 8-position. J. Am. Chem. Soc. 133, 3780-3783 (2011).

14 Konishi, S. et al. Site-selective C-H borylation of quinolines at the $\mathrm{C} 8$ position catalyzed by a silica-supported phosphane-iridium system. Chem. Asian. J. 9, 434-438 (2014).

15 Wang, P. et al. Ligand-accelerated non-directed C-H functionalization of arenes. Nature 551, 489-493 (2017).

16 Murai, M., Nishinaka, N. \& Takai, K. Iridium-catalyzed sequential silylation and borylation of heteroarenes cased on regioselective $\mathrm{C}-\mathrm{H}$ bond activation. Angew. Chem. Int. Ed. 57, 5843-5847 (2018).

17 Lou, S.-J. et al. Regiodivergent C-H alkylation of quinolines with alkenes by half-sandwich rare-earth catalysts. J. Am. Chem. Soc. 142, 18128-18137 (2020).

18 Leow, D., Li, G., Mei, T.-S. \& Yu, J.-Q. Activation of remote meta-C-H bonds assisted by an end-on template. Nature 486, 518-522 (2012).

19 Kuninobu, Y., Ida, H., Nishi, M. \& Kanai, M. A meta-selective C-H borylation directed by a secondary interaction between ligand and substrate. Nat. Chem. 7, 712-717 (2015).

20 Bag, S. et al. Remote para-C-H functionalization of arenes by a D-shaped biphenyl template-based assembly. J. Am. Chem. Soc. 137, 11888-11891 (2015).

21 Hoque, M. E., Bisht, R., Haldar, C. \& Chattopadhyay, B. Noncovalent interactions in Ir-catalyzed C-H activation: L-shaped ligand for para-selective borylation of aromatic esters. J. Am. Chem. Soc. 139, 7745-7748 (2017).

22 Yang, L., Uemura, N. \& Nakao, Y. Meta-selective C-H borylation of benzamides and pyridines by an iridium-Lewis acid bifunctional catalyst. J. Am. Chem. Soc. 141, 7972-7979 (2019).

23 Reyes, R. L. et al. Asymmetric remote C-H borylation of aliphatic amides and esters with a modular iridium catalyst. Science 369, 970-974 (2020).

24 Meng, G. et al. Achieving site-selectivity for $\mathrm{C}-\mathrm{H}$ activation processes based on distance and geometry: A carpenter's approach. J. Am. Chem. Soc. 142, 1057110591 (2020).

25 Zhang, Z., Tanaka, K. \& Yu, J.-Q. Remote site-selective C-H activation directed by a catalytic bifunctional template. Nature 543, 538-542 (2017).

26 Ramakrishna, K. et al. Coordination assisted distal C-H alkylation of fused heterocycles. Angew. Chem. Int. Ed. 58, 13808-13812 (2019).

27 Shi, H. et al. Differentiation and functionalization of remote $\mathrm{C}-\mathrm{H}$ bonds in adjacent positions. Nat. Chem. 12, 399-404 (2020). 
28 Chu, L. et al. Remote meta-C-H activation using a pyridine-based template: achieving site-selectivity via the recognition of distance and geometry. ACS Cent. Sci. 1, 394-399 (2015).

29 Lerchen, A. et al. Non-directed cross-dehydrogenative (hetero)arylation of allylic $\mathrm{C}\left(\mathrm{sp}^{3}\right)-\mathrm{H}$ bonds enabled by $\mathrm{C}-\mathrm{H}$ activation. Angew. Chem. Int. Ed. 57, 15248-15252 (2018).

30 Fu, L., Zhang, Z., Chen, P., Lin, Z. \& Liu, G. Enantioselective copper-catalyzed alkynylation of benzylic C-H bonds via radical relay. J. Am. Chem. Soc. 142, 12493-12500 (2020).

31 Porey, S. et al. Alkyne linchpin strategy for drug: pharmacophore conjugation: experimental and computational realization of a meta-selective inverse sonogashira coupling. J. Am. Chem. Soc. 142, 3762-3774 (2020).

32 Pan, P. et al. Structure-based drug design and identification of $\mathrm{H}_{2} \mathrm{O}$-soluble and low toxic hexacyclic camptothecin derivatives with improved efficacy in cancer and lethal inflammation models in vivo. J. Med. Chem. 61, 8613-8624 (2018).

33 Krajewska, J., Olczyk, T. \& Jarzab, B. Cabozantinib for the treatment of progressive metastatic medullary thyroid cancer. Expert Rev. Clin. Pharmacol. 9, 69-79 (2016).

34 Personeni, N., Rimassa, L., Pressiani, T., Smiroldo, V. \& Santoro, A. Cabozantinib for the treatment of hepatocellular carcinoma. Expert Rev. Anticancer Ther. 19, 847-855 (2019).

35 Hwang, J. Y. et al. Synthesis and evaluation of 7-substituted 4-aminoquinoline analogues for antimalarial activity. J. Med. Chem. 54, 7084-7093 (2011). 